\title{
Communication: Enhancement of dopant dependent x-ray photoelectron spectroscopy peak shifts of Si by surface photovoltage
}

Hikmet Sezen, and Sefik Suzer

Citation: The Journal of Chemical Physics 135, 141102 (2011);

View online: https://doi.org/10.1063/1.3652964

View Table of Contents: http://aip.scitation.org/toc/jcp/135/14

Published by the American Institute of Physics

\section{Articles you may be interested in}

Transient surface photovoltage in $\mathrm{n}$ - and $\mathrm{p}-\mathrm{GaN}$ as probed by $\mathrm{x}$-ray photoelectron spectroscopy Applied Physics Letters 98, 111901 (2011); 10.1063/1.3564892

Growth of native oxide on a silicon surface

Journal of Applied Physics 68, 1272 (1998); 10.1063/1.347181

New ambient pressure photoemission endstation at Advanced Light Source beamline 9.3.2

Review of Scientific Instruments 81, 053106 (2010); 10.1063/1.3427218

Investigation on thermal evaporated $\mathrm{CH}_{3} \mathrm{NH}_{3} \mathrm{Pbl}_{3}$ thin films

AIP Advances 5, 097111 (2015); 10.1063/1.4930545

A high-order harmonic generation apparatus for time- and angle-resolved photoelectron spectroscopy Review of Scientific Instruments 84, 075106 (2013); 10.1063/1.4812992

Communication: Multicanonical entropy-like solution of statistical temperature weighted histogram analysis method

The Journal of Chemical Physics 135, 141101 (2011); 10.1063/1.3651627

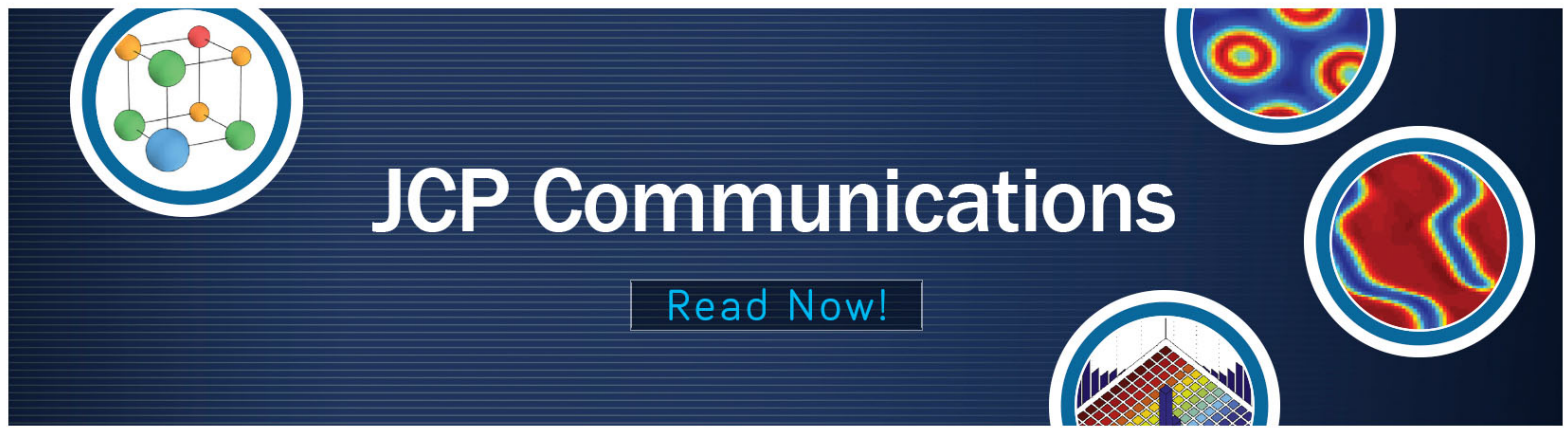




\title{
Communication: Enhancement of dopant dependent x-ray photoelectron spectroscopy peak shifts of Si by surface photovoltage
}

\author{
Hikmet Sezen and Sefik Suzer ${ }^{\text {a) }}$ \\ Department of Chemistry, Bilkent University, 06800 Ankara, Turkey
}

(Received 5 August 2011; accepted 28 September 2011; published online 10 October 2011)

\begin{abstract}
Binding energies measured by $\mathrm{x}$-ray photoelectron spectroscopy (XPS) are influenced by doping, since electrons are transferred to (p-type) and from (n-type) samples when they are introduced into the spectrometer, or brought into contact with each other (p-n junction). We show that the barely measurable Si2p binding energy difference between moderately doped $\mathrm{n}$ - and $\mathrm{p}$-Si samples can be enhanced by photoillumination, due to reduction in surface band-bending, which otherwise screens this difference. Similar effects are also measured for samples containing oxide layers, since the bandbending at the buried oxide-Si interfaces is manifest as photovoltage shifts, although XPS does not probe the interface directly. The corresponding shift for the oxide layer of the $\mathrm{p}$-Si is almost twice that of without the oxide, whereas no measurable shifts are observable for the oxide of the n-Si. These results are all related to band-bending effects and are vital in design and performance of photovoltaics and other related systems. @ 2011 American Institute of Physics. [doi:10.1063/1.3652964]
\end{abstract}

It is well established that XPS binding energies of the core-levels of $\mathrm{n}$ - and $\mathrm{p}$-doped $\mathrm{Si}$ and other semiconductors such as GaAs exhibit measurable shifts, which have been used for spectroscopic analyses ${ }^{1-6}$ and/or spectromicroscopic imaging $^{7-16}$ in numerous materials, as well as across p-n junctions in various devices. However, these shifts are significantly influenced/screened by the presence of oxide or contaminant interfaces as well as due to surface band-bending. Hence dis-entanglement of contribution of the various effects is challenging and desirable. Since XPS is inherently a surface sensitive technique (probe depth of 1-10 nm), photovoltage or surface photovoltage is another process which contributes significantly in a negative sense to the measured XPS binding energy (BE) shifts by screening them. On the other hand, understanding and intelligent use of the response of various semiconductor structures to light is vital in numerous applications such as manufacturing detectors, sensors, photocells, photovoltaic, and photo-catalytic devices, scientific and technical instruments, etc., which has led to extensive scientific research, especially in recent years. ${ }^{17-21}$

As a continuation of our efforts in probing the photovoltage phenomenon, ${ }^{22,23}$ in this contribution, we will present certain experimental results related to XPS binding energy shifts without and under photoillumination of $\mathrm{n}$ - and $\mathrm{p}$-doped silicon samples separately, as well as together, connected in an ad hoc p-n junction, where we show that the surface photovoltage partially offsets the band-bending shifts and allows one to perform measurements at close to flat-band condition. The band-bending manifests in different fashions by introduction of oxide layers, since now the additional semiconductoroxide interfaces have to be taken into account also. However, the overall effect of the photoillumination is still operative

\footnotetext{
a) Author to whom correspondence should be addressed. Electronic mail: suzer@fen.bilkent.edu.tr.
}

and leads to enhancement of dopant-induced binding energy shifts.

Commercially available $\mathrm{n}$ - and p-doped $\mathrm{Si}(100)$ wafers, with their rated conductivities of $10-20$ and $28-31 \Omega \mathrm{cm}$, respectively, are cleaned with HF before analysis. The same samples are also analyzed after growing $>15 \mathrm{~nm}$ oxide layers by heating at $700{ }^{\circ} \mathrm{C}$ in air for $\sim 3 \mathrm{~h}$. A Thermo Fisher $\mathrm{K}$-Alpha electron spectrometer with monochromatic $\mathrm{AlK} \alpha$ $\mathrm{x}$-rays is used for XPS analysis with an overall resolution better than $0.30 \mathrm{eV}$. The intensity of $\mathrm{x}$-ray photons focused on the surface was on the order of $10^{12}$ photons $/ \mathrm{cm}^{2} \mathrm{~s}$. For measurement of the binding energy differences the precision is estimated to be better than $0.03 \mathrm{eV}$ for the $\mathrm{Si} 2 \mathrm{p}$ of the $\mathrm{n}$ - and $\mathrm{p}-\mathrm{Si}$, but the peaks become much broader for the oxide, hence the precision is decreased to around $0.06 \mathrm{eV}$. The photovoltage measurements were performed using four different lasers at $980,635,532$, and $405 \mathrm{~nm}$ in the C.W. mode, as the excitation probe with rated power of $>50 \mathrm{~mW}$. A shutter connected to a signal generator was used to turn the laser on and off periodically.

For commonly used doped $\mathrm{Si}$ semiconductor samples, the chemical composition of the dopants $\left(\sim 1: 10^{8}\right)$ is well below the detection limit of the present commercial XPS instruments $(\sim 0.1 \mathrm{a} / \mathrm{o})$. However, since the position of XPS peaks is measured and/or corrected with respect to the Fermi-level of the sample under investigation, dopant related states within the bandgap dictate the pinning of the Fermi-level, hence causing measurable binding energy shifts as depicted schematically in Figure 1(a) for $\mathrm{Si}$. Using the rated conductivities, we estimate that $0.53 \mathrm{eV}$ binding energy difference should be found between the Si2p, as well as other core and valence levels of our $\mathrm{n}$ - and $\mathrm{p}$-Si (see supplementary material ${ }^{24}$ for estimation of this value), as opposed to only $0.18 \mathrm{eV}$ measured, as shown in Figure 2 (and Figure S1, supplementary material ${ }^{24}$ ) for a pseudo Si p-n junction, artificially created by connecting the $\mathrm{n}$ - and $\mathrm{p}$-Si via a gold metal strip. This difference is attributed 
(a)

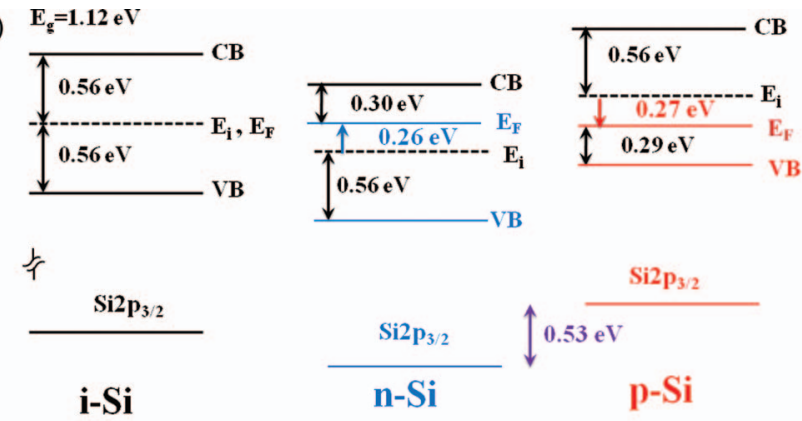

(b)

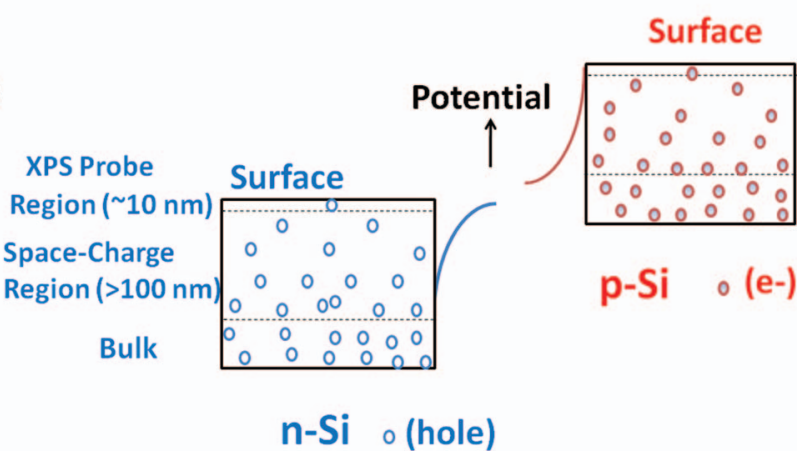

FIG. 1. (a) Schematic energy level diagrams of intrinsic, $n-$ and p-doped Si samples used. (b) Schematic diagrams of the n- and p-doped Si showing the variation in the hole and the electron distributions from the surface down towards the bulk. For the n-sample the surface region has gradually reduced number of holes across the space-charge region $(\sim 1000 \mathrm{~nm})$, and the opposite is operative for the p-sample. Note that only the top $\sim 10 \mathrm{~nm}$ is probed by XPS, hence it can be safely assumed that the potential variation is constant in this region.

to the effective band-bending as also shown schematically in the same figure. ${ }^{25-27}$

A simplified and more chemical interpretation is as follows. An n-doped sample is electrically neutral but has more or less free and delocalized electrons, while a p-doped sample has the opposite (i.e., holes). Upon contact with a metal (or the XPS spectrometer), a certain number of electrons are transferred from the $\mathrm{n}$ - to the p-sample via the Au-metal strip (or through the spectrometer ground connection), until the electrochemical potentials are equalized, but resulting in positively charged $\mathrm{n}$ - and negatively charged $\mathrm{p}-\mathrm{Si}$ as shown schematically in Figure 1(b). Accordingly, the kinetic energy of the photoelectrons leaving the $n$ - is reduced (hence the binding energy is increased) and those of $\mathrm{p}$ - are increased (BE decreased). However, this scheme is valid only for the bulk, and the electrical potentials are once more modified from the surface towards the bulk due to the band-bending, resulting in a space-charge region.

As also depicted in Figure 2, one way to reduce the band-bending and to approach the flat-band condition is photoillumination, which decreases the band-bending due to the surface photovoltage created, and for our experimental conditions with the $405 \mathrm{~nm}(3.1 \mathrm{eV}) \mathrm{cw}$ laser (and others as well), results in a factor of 3 enhanced $(0.49 \mathrm{eV})$ binding energy difference between the $\mathrm{Si} 2 \mathrm{p}$ of the $\mathrm{n}$ - and $\mathrm{p}$-sides, which is much closer to the estimated value of $0.53 \mathrm{eV}$. Furthermore, and as depicted in Figure 3, this difference can be achieved with any laser source with reasonable power

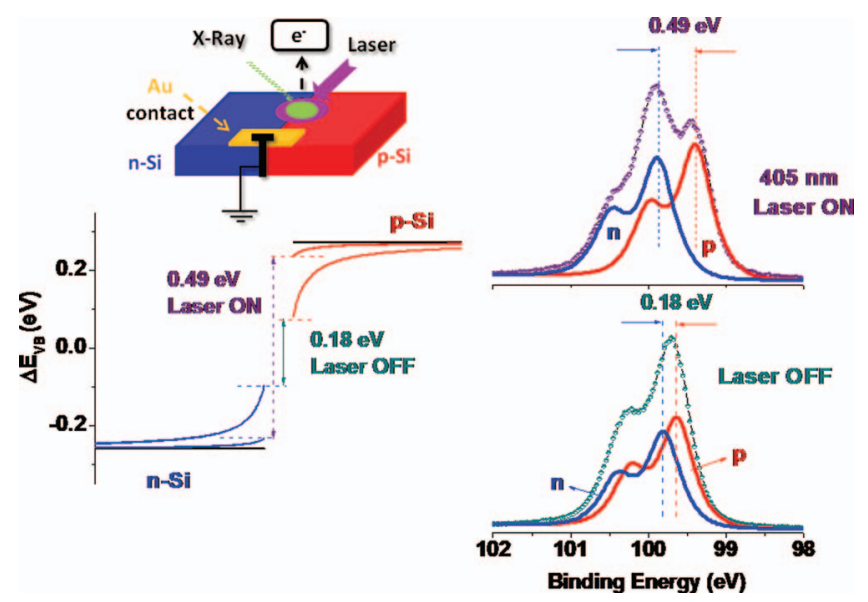

FIG. 2. Compound Si2p level of an ad hoc p-n junction, created artificially, without and under $405 \mathrm{~nm}$ laser illumination. Without the illumination of the $\mathrm{Si} 2 \mathrm{p}$ spin-orbit doublet displays a shoulder on the low energy side and is slightly broader (see also Figure S1), and only after peak deconvolution can the difference between the $n$ - and p- be realized. However, under illumination a clear indication of at least 3 components ( 2 are still overlapping) are visible, and the difference is increased by a factor of 3 . The inset schematically shows the experimental set-up.

(>50 mWatt), in the NIR-Visible range, since the Si bandgap is only $1.12 \mathrm{eV}$. Similar observations were also recorded (not shown) for other low bandgap systems such as $\mathrm{Ge}\left(\mathrm{E}_{\mathrm{g}}\right.$ $=0.68 \mathrm{eV})$, and GaAs $\left(\mathrm{E}_{\mathrm{g}}=1.42 \mathrm{eV}\right){ }^{28}$ Note that, upon illumination the spectroscopic resolving power between the $\mathrm{n}$ - and $\mathrm{p}-\mathrm{Si}$ becomes overwhelmingly increased due to the photovoltage operating in opposite directions.

A simple and chemical picture can be provided as follows. Upon illumination with light having energy greater than the bandgap, extra electron-hole pairs are created in the semiconductor. For the $\mathrm{n}-\mathrm{Si}$, the extra electrons are repelled away from the surface by the built-in potential due to band-bending, and conversely the holes are trapped near the surface, and the opposite is operative for the $\mathrm{p}$-Si, with the net result of flattened bands on both sides. 5,6

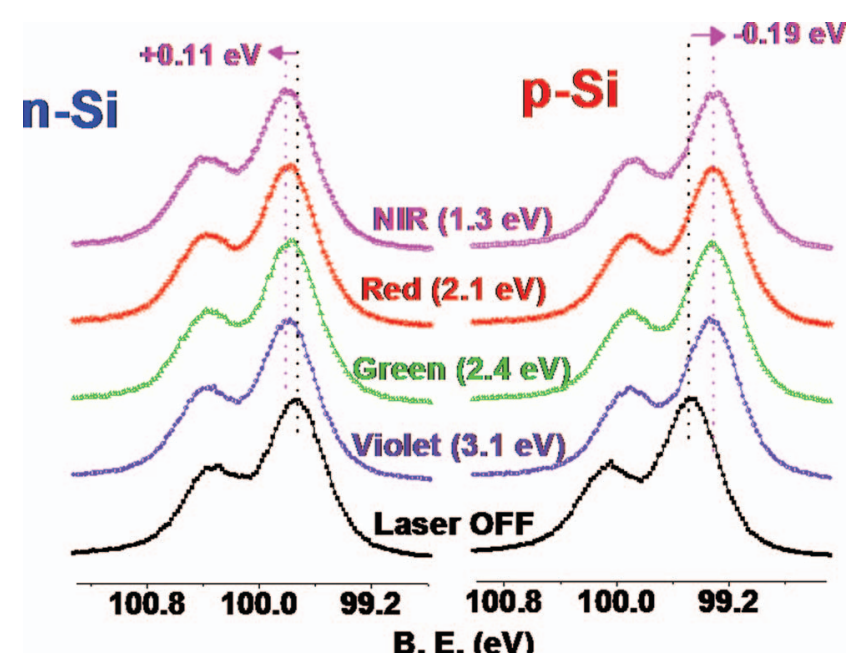

FIG. 3. Si2p peaks of the separate $n$ - and $p$-samples recorded under different laser illuminations. 


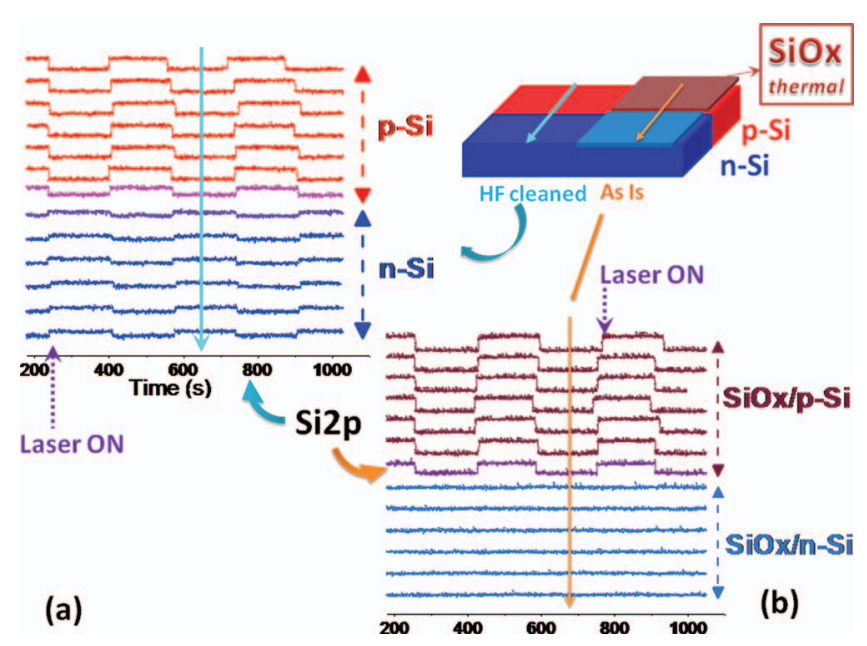

FIG. 4. Variations in the positions of the Si2p peak of the ad hoc p-n junction under periodic laser illumination as the sampling position is moved 5 $\mathrm{mm}$ across the junction in $0.5 \mathrm{~mm}$ steps, and the probed area is $\sim 400 \mu \mathrm{m}$ in diameter. (a) HF-cleaned $\mathrm{Si}$ samples (Si2p peaks is around $99 \mathrm{eV}$ ), and (b) samples containing $>15 \mathrm{~nm}$ thermal oxide layer (around $104 \mathrm{eV}$ ). The inset schematically shows the experimental details.

Although we deliberately connected our samples to mimic a p-n junction, the observation we are reporting is not actually related to the potential variations across the junction, as reported by previous workers, ${ }^{4-6}$ but are simply related only to their chemical modifications introduced by the dopants, since they are also observed when the samples are analyzed separately as, for example, shown in Figure 3. To illustrate this point further we have performed a line-scan consisting of a series of measurements covering large distances (compared to the width of a p-n junction) on both sides of the junction as shown in Figure 4(a). As revealed by the figure, the observed binding energy changes with laser illumination are constant throughout one region, reproducible and reversible throughout the entire surface of the $\mathrm{p}$ - and the n-samples, and exhibiting constant shifts in opposite directions; thus proving that these changes are related to changes in the surface band-bending by the action of light.

Similar measurements are also performed for the same system after growing $>15 \mathrm{~nm}$ oxide layers on both samples as depicted in Figure 4(b), with a somewhat surprising result, where we observe a larger photoinduced binding energy shift in the p- but no measurable one for the n-side. Such differences must be attributed to the additional oxidesemiconductor interfaces introduced. It is well known and established that electrons are injected from the oxide to the silicon so that the oxide side of the interface is positively charged for a p-type silicon, ${ }^{25-27}$ as shown schematically in Figure 5(a). Hence the overall band-bending is additive (p-type + oxide), resulting in a larger shift upon illumination. The absence of any measurable shift for the n-sample must follow the same argument, and to be the result of near cancellation of the surface upward bending of the $\mathrm{n}$-Si by the oxide downward bending at the interface, as schematically shown in Figure 5(b).

It is also very surprising that the overall photovoltage binding energy shift between the $\mathrm{p}$ - and $\mathrm{n}-\mathrm{Si}$ is more or less

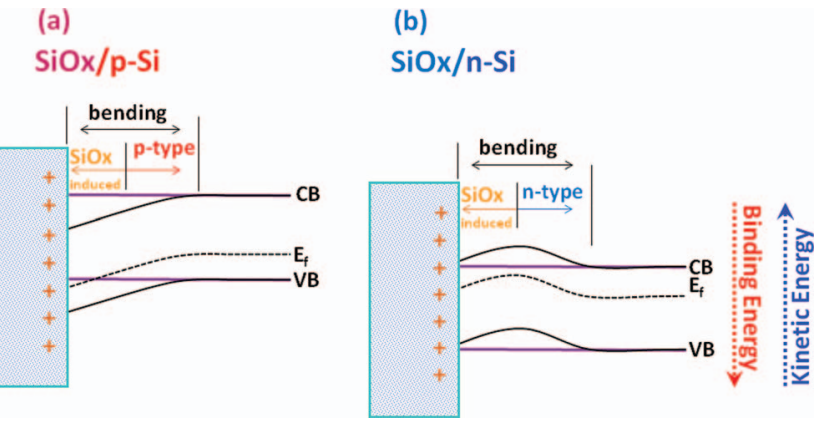

FIG. 5. Schematic representation of the variations in the band-bending at the oxide-Si interface for (a) p- and (b) n-Si.

conserved; i.e., the combined upward shift of the $\mathrm{p}-\mathrm{Si}(+0.22)$ and the downward shift of the $\mathrm{n}-\mathrm{Si}(-0.09)$ is not drastically different from the observed upward shift of the oxide/p-Si $(+0.36 \pm 0.06 \mathrm{eV})$. Note that the Si2p of the oxide layers are inherently broader, hence carry larger uncertainty. Furthermore, since the probe length of the XPS signal is smaller than oxide thickness, the interfaces are not probed (buried) directly. But their photovoltage shifts are faithfully translated, since the penetration depth of all the photons we used are larger than the oxide layer thickness, and are able to fully flatten the interface(s).

In summary, we show that for $\mathrm{Si}$, the dopant dependent shifts in the positions of XPS peaks can be enhanced by illumination with light having energy above its bandgap, hence can be conveniently used for determining the type of doping in a chemically addressed fashion. Furthermore, such shifts are also operative for the samples containing oxide layers, which, in addition, provide information about the presence and/or the character of the buried oxide-semiconductor interfaces. Extension of this work will follow for assessing the generality of our findings to more complicated and naturally doped semiconductors such as $\mathrm{CdS}, \mathrm{TiO}_{2}$, etc., and/or composite systems and interfaces.

This work was partially supported by TUBA (the Turkish Academy of Sciences). We are also grateful to Professor Dr. Mehmet Erbudak (ETH, Zurich) and Professor Dr. Oguz Gulseren (Bilkent, Ankara) for their fruitful discussions.

${ }^{1}$ F. G. Allen and G. W. Gobeli, Phys. Rev. 127, 150 (1962).

${ }^{2}$ J. Hedman, Y. Baer, A. Berndtsson, M. Klasson, G. Leonhardt, R. Nilsson, and C. Nordling, J. Electron Spectrosc. Relat. Phenom. 1, 101 (1972).

${ }^{3}$ F. Himpsel, G. Hollinger, and R. Pollak, Phys. Rev. B 28, 7014 (1983).

${ }^{4}$ J. E. Demuth, W. J. Thompson, N. J. DiNardo, and R. Imbihl, Phys. Rev. Lett. 56, 1408 (1986).

${ }^{5}$ J. Long, H. Sadeghi, J. Rife, and M. Kabler, Phys. Rev. Lett. 64, 1158 (1990).

${ }^{6}$ J. Long and V. Bermudez, Phys. Rev. B 66, 121308 (2002).

${ }^{7}$ M. Giesen, R. J. Phaneuf, E. D. Williams, T. L. Einstein, and H. Ibach, Appl. Phys. A 64, 423 (1997).

${ }^{8}$ M. Marsi, L. Casalis, L. Gregoratti, S. Gunther, A. Kolmakov, J. Kovac, D. Lonza, and M. Kiskinova, J. Electron Spectrosc. Relat. Phenom. 84, 73 (1997).

${ }^{9}$ R. J. Phaneuf, H. C. Kan, M. Marsi, L. Gregoratti, S. Gunther, and M. Kiskinova, J. Appl. Phys. 88, 863 (2000).

${ }^{10}$ V. W. Ballarotto, K. Siegrist, R. J. Phaneuf, E. D. Williams, and S. Mogren, Surf. Sci. 461, L570 (2000). 
${ }^{11}$ P. Siffalovic, M. Drescher, and U. Heinzmann, Europhys. Lett. 60, 924 (2002).

${ }^{12}$ S. Heun, S. Kremmer, D. Ercolani, H. Wurmbauer, and C. Teichert, J. Electron Spectrosc. Relat. Phenom. 144, 1163 (2005).

${ }^{13}$ L. Frank, I. Mullerova, D. A. Valdaitsev, A. Gloskovskii, S. A. Nepijko, H. J. Elmers, and G. Schonhense, J. Appl. Phys. 100, 5 (2006).

${ }^{14}$ J. Charlier, S. Palacin, J. Leroy, D. Del Frari, L. Zagonel, N. Barrett, O. Renault, A. Bailly, and D. Mariolle, J. Mater. Chem. 18, 3136 (2008).

${ }^{15}$ N. Barrett, L. F. Zagonel, O. Renault, and A. Bailly, J. Phys. Condens. Matter 21, 314015 (2009).

${ }^{16}$ F. de la Pena, N. Barrett, L. F. Zagonel, M. Walls, and O. Renault, Surf. Sci. 604, 1628 (2010)

${ }^{17}$ L. Kronik and Y. Shapira, Surf. Sci. Rep. 37, 1 (1999).

${ }^{18}$ A. Schellenberger, R. Schlaf, C. Pettenkofer, and W. Jaegermann, Phys. Rev. B 45, 3538 (1992).
${ }^{19}$ R. Schlaf, A. Klein, C. Pettenkofer, and W. Jaegermann, Phys. Rev. B 48, 14242 (1993).

${ }^{20}$ M. Foussekis, J. D. Ferguson, A. A. Baski, H. Morkoc, and M. A. Reshchikov, Physica B 404, 4892 (2009).

${ }^{21}$ M. A. Reshchikov, M. Foussekis, and A. A. Baski, J. Appl. Phys. 107, 113535 (2010).

${ }^{22}$ H. Sezen and S. Suzer, Surf. Sci. 604, L59 (2010)

${ }^{23}$ H. Sezen, E. Ozbay, O. Aktas, and S. Suzer, Appl. Phys. Lett. 98, 111901 (2011).

${ }^{24}$ See supplementary material at http://dx.doi.org/10.1063/1.3652964 for estimation of this value.

${ }^{25}$ C. Munakata, S. Nishimatsu, N. Honma, and K. Yagi, Jpn. J. Appl. Phys., Part 1 23, 1451 (1984).

${ }^{26}$ W. M. Lau, J. Appl. Phys. 65, 2047 (1989).

${ }^{27}$ T. J. Sarapatka, Surf. Sci. 275, 443 (1992).

${ }^{28}$ J. D. Plummer, M. D. Deal, and P. B. Griffin, Silicon VLSI Technology: Fundamentals, Practice and Modeling (Prentice-Hall, New Jersey, 2000). 